\title{
Clima laboral en una escuela de medicina. Estudio de seguimiento
}

\author{
Ignacio Sánchez D ${ }^{1}$, G regorio Airola G 2a, Tatiana Cayazzo A 2b, \\ Nuria Pedrals $\mathrm{G}^{3 \mathrm{~b}}$, Magdalena Rodríguez $\mathrm{M}^{3 \mathrm{a}}$, \\ Luis Villarroel $\mathrm{D}^{4 c}$. \\ Work environment assessment \\ at a medical school
}

Background: The work environment of an organization has to do with a set of permanent qualities that are experienced by its members. Aim: To assess the work environment perception of faculty members of a Medical School in two different periods (2005 and 2007). Material and methods: A standardized survey was applied to faculty members and Departament chairs of the academic units of our Medical School. The survey used the Likert scale from 1 to 5 and included 59 items, distributed in 8 factors. Additionally, there were two groups of statements, indicating the priorities for the School and for the academic departments. Results: In the study performed in year 2005, the survey was answered by a total of 399 faculty members (68\%) and in year 2007, it was answered by 408 members (68\%). The global climate perceptions were $66 \%$ and $68 \%$ in 2005 and 2007, respectively (ns). Among the dimensions evaluated, communications $(p=0,62)$ and physical conditions $(p=0,008)$ improved in the two years period. Conclusions: The applied instrument was stable and useful to knowing the requirements of the faculty members and for improving the organizational climate (Rev Méd Chile 2009; 137: 1427-36).

(Key words: Burnout, professional; Educational assessment; Faculty, medical)

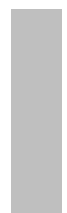

Recibido el 19 de enero, 2009. Aceptado el 16 de septiembre, 2009.

${ }^{1}$ Decanato, ${ }^{2}$ Gerencia de Personal, ${ }^{3}$ Subdirección Administrativa, ${ }^{4}$ Departamento de Salud Pública, Facultad de Medicina, Pontificia Universidad Católica de Chile.

angeniero Comercial

bPsicóloga

${ }^{\mathrm{C}} \mathrm{PhD}$ Estadística

L a medicina académica constituye un ambiente de trabajo único, en que interactúan miembros de generaciones diferentes y donde estos equipos son fundamentales para cumplir la misión, particularmente la de educar, investigar y crear nuevo conocimiento. Esto puede generar un aumento en el conocimiento y actividad académica, pero también conflictos ya que cada generación tiene

Correspondencia a: Dr. Ignacio Sánchez D. Decanato, Facultad de Medicina, Pontificia Universidad Católica de Chile. Lira 44, piso 2, Santiago, Chile. Fono: 354 6422. Fax: 638 6520. E mail: igsan@med.puc.cl diferentes valores y expectativas. Las publicaciones que abordan las descripciones de las diferentes generaciones y sus conflictos en el ámbito laboral se aplican claramente al ámbito de la medicina académica ${ }^{1-3}$.

A pesar de que existe satisfacción y bienestar entre los académicos de las escuelas de medicina, los crecientes factores generadores de estrés y descontento son un real desafío, por lo que debemos encontrar diversas formas de apoyar y motivar a los académicos en su desarrollo personal y profesional a través de programas de consejería y desarrollo académico centrados en los más jóvenes ${ }^{4,5}$. 
Según la información que tenemos, no existen estudios en Chile que describan las respuestas de un número importante de académicos, de diversas instituciones y disciplinas en relación a las condiciones laborales del ambiente académico. Un estudio extranjero evaluó el grado de satisfacción laboral y personal, y el nivel de estrés laboral de voluntarios y docentes de jornada completa en una escuela de medicina. Para muchos académicos, el atractivo de la academia estuvo en la variedad de la actividad docente, investigación y asistencia, sin embargo, se describen importantes fuentes de tensión en el trabajo, lo que presenta desafíos singulares 6 .

En la definición de clima laboral no existe unanimidad, ya que no hay atributos permanentes, y en el largo plazo, la cultura organizacional puede incorporar estos valores. La definición del término varía desde factores organizacionales objetivos (estructura, políticas y reglas), hasta atributos completamente subjetivos (opiniones, cordialidad, apoyo, etc.). Las siguientes definiciones se han planteado en clima laboral: "El conjunto de características permanentes que describen una organización, la distingue de otra e influye en el comportamiento de las personas que la forman" (Forehand y Gilmer, 1964); "Los efectos subjetivos, percibidos del sistema formal, de estilo informal de liderazgo y otros factores ambientales importantes sobre las actitudes, creencias, valores y motivaciones de las personas que trabajan en una organización" (Ltiwin y Stringer, 1968); y "Representa las percepciones que el individuo tiene de la organización para la cual trabaja y la opinión que se haya formado de ella en términos de autonomía, estructura, recompensas" (Dessler, 1979 y 1993). Las perspectivas descritas pueden clasificarse en 3 categorías: 1) Aquellos que consideran al clima organizacional como variable independiente (un factor que influye en la satisfacción); 2) como variable dependiente (al variar algunos factores se influye en el clima organizacional) y 3) como variable interpuesta (clima organizacional actúa de enlace entre factores como estructura de rendimiento).

Nuestro objetivo fue identificar las percepciones que tienen los académicos en relación a las características laborales de la Escuela de Medicina de la Pontificia Universidad Católica de Chile en 2 períodos (año 2005 y año 2007) y apoyar la gestión de los Departamentos y de la Escuela, proporcionando información relevante que pueda colaborar en la evaluación y planificación de las actividades académicas.

\section{MATERIAL Y MÉTODO}

La muestra del estudio se obtuvo mediante una encuesta, previamente elaborada y validada como instrumento de medición. En la etapa de diseño, se procedió a definir las dimensiones a evaluar, se construyeron los ítems, se aprobó el instrumento de medición y se diseñó la metodología de aplicación. En la creación del instrumento, se conformó un grupo interdisciplinario de académicos, profesionales administrativos y de gestión (médicos, ingenieros comerciales y psicólogos), que revisaron y adaptaron a la realidad de la Escuela de Medicina afirmaciones de diversos instrumentos, tales como Great Place to Work, Stinger (2002); Bloch y Whiteley (2003), Likert (1961 y 1976) y Larraín (1976). De esta forma, se redactó un instrumento sencillo y comprensible para todas las personas.

La encuesta se aplicó a los académicos de la Escuela de Medicina en diciembre de 2005 y 2007 en forma colectiva, distribuyéndose la encuesta a cada jefe para ser entregada a los académicos de sus respectivos departamentos. Se hizo entrega de una encuesta y hoja de respuesta por persona, siendo esta última devuelta en un sobre cerrado a la Gerencia de Personal (el contenido de la encuesta se encuentra disponible para los lectores que lo requieran en decano@med.puc.cl).

El instrumento aplicado utilizó la escala Likert de 1 a 5 e incluyó 59 ítems, distribuidos en 8 factores, además de una pregunta final acerca de la relevancia que las personas le asignan a cada una de las dimensiones evaluadas. Los factores evaluados y las percepciones asociadas se presentan en la Tabla 1. A través de una pregunta final de satisfacción global con la organización, se buscó medir la percepción general. En la tabulación del instrumento, sus resultados se presentaron sin identificar a las personas en forma individual. Con el objetivo de mantener la confidencialidad de las respuestas, cuando la segmentación representó menos de cuatro personas, no se mostraron los resultados para que éstas no pudieran ser identificadas, no obstante, sus resulta- 
Tabla 1. Factores evaluados y percepciones asociadas

\begin{tabular}{|c|c|}
\hline Factor evaluado & Percepción asociada \\
\hline Motivación & $\begin{array}{l}\text { Importancia del trabajo realizado e interés por realizarlo, la permanen- } \\
\text { cia en la organización, respeto y desarrollo personal }\end{array}$ \\
\hline Recompensa & $\begin{array}{l}\text { Salario adecuado de acuerdo a condiciones de mercado y apropiado al } \\
\text { trabajo desempeñado. Utilidad de beneficios }\end{array}$ \\
\hline Relaciones interpersonales & Clima de trabajo grato, confianza y valoración entre las personas \\
\hline Comunicación & Claridad de la información vertical y horizontal \\
\hline Materiales y equipos & $\begin{array}{l}\text { Conveniencia de los materiales, equipos y condiciones ambientales (luz, } \\
\text { ventilación, seguridad, etc.) }\end{array}$ \\
\hline Lider & Adecuación técnica y humana de las jefaturas \\
\hline Imparcialidad & Trato justo e igualdad de oportunidades para desarrollarse en el cargo \\
\hline
\end{tabular}

dos fueron considerados para la evaluación general. Se realizó un análisis de datos según estadística descriptiva y, cualitativamente, se consideraron los porcentajes de respuestas que correspondían a los rangos 4 y 5 de la encuesta ("de acuerdo" y "completamente de acuerdo") en cada afirmación. Es decir, sólo se consideraron aquellas respuestas en que los académicos concondaron con la afirmación realizada en la pregunta, encontrando que la institución se desempeña bien o muy bien en esa materia. Para efecto de análisis y conclusiones, se definió una escala de cuatro categonías, según los porcentajes de satisfacción, los que fueron: menor a $50 \%$ (se consideró Deficiente), entre 50,1\% y 65\% (Regular); entre 65,1 y $70 \%$ (Bueno) y mayor a 70,1\% (Muy Bueno).

En ambas ocasiones, los resultados globales fueron presentados primeramente al conjunto de los Jefes de Departamentos. Luego, éstos se reunieron al interior de las unidades, realizando un diagnóstico local y acordando líneas de trabajo a seguir. La presentación de los resultados obtenidos en una encuesta de clima laboral se considera una intervención en sí misma, ya que favorece que el grupo se haga cargo colectivamente de un diagnóstico y de las fortalezas y debilidades como grupo acordando acciones a desarrollar. En esta etapa, las jefaturas utilizaron distintas metodologías, algunas trabajaron intragrupos, otras optaron por acompañamiento personal asesorados por la Gerencia de Recursos Humanos y algunos departamentos desarrollaron Jornadas de Planificación para generar una visión compartida de la Misión y fortalecer el trabajo en equipo del Departamento (ver Tablas 2 y 3). Debido a que los instrumentos de clima laboral aplicados son anónimos, con la

Tabla 2. M etodología de trabajo

\begin{tabular}{|ll|}
\hline$N^{0}$ & Etapas \\
\hline 1 & $\begin{array}{l}\text { Taller de Trabajo (12 h aprox.) } \\
2\end{array}$ \\
$\begin{array}{l}\text { Análisis de los Trabajos: el equipo de consultores preparará una síntesis de los diferentes } \\
\text { trabajos realizados durante este taller }\end{array}$ \\
3 & $\begin{array}{l}\text { Definiciones del Jefe de Departamento: revisión de documento con distintos integrantes del } \\
\text { departamento }\end{array}$ \\
4 & Presentación de Trabajo: presentación del documento final \\
\hline
\end{tabular}


Tabla 3. Taller de trabajo

\begin{tabular}{|c|c|c|}
\hline H orario & Actividad & Descripción \\
\hline $15: 00-15: 30$ & \multicolumn{2}{|l|}{$\begin{array}{l}\text { Presentación de expectativas } \\
\text { y objetivos }\end{array}$} \\
\hline \multirow{4}{*}{$15: 30-17: 00$} & \multirow[t]{4}{*}{ Trabajo en equipo } & Concepto de trabajo en equipo \\
\hline & & Dinámica grupal \\
\hline & & Estructura de los equipos \\
\hline & & Etapas de formación y riesgos \\
\hline \multirow[t]{3}{*}{$17: 30-19: 00$} & \multirow[t]{3}{*}{ ¿En qué creemos? } & Trabajo individual \\
\hline & & Trabajo grupal \\
\hline & & Plenarias y conclusiones \\
\hline \multicolumn{3}{|c|}{ Día 2} \\
\hline \multirow[t]{6}{*}{ 08:30 - 10:30 } & \multirow[t]{6}{*}{ Entorno } & Conceptos claves \\
\hline & & Análisis del entorno: \\
\hline & & - Análisis de la industria y tendencias \\
\hline & & - Diagnóstico entorno \\
\hline & & Trabajo individual y grupal \\
\hline & & Presentaciones \\
\hline \multirow[t]{3}{*}{$11: 00-12: 30$} & \multirow[t]{3}{*}{ Misión y visión } & Concepto: Definición de misión - visión \\
\hline & & Misión: Trabajo individual y grupal \\
\hline & & Presentaciones \\
\hline \multirow[t]{4}{*}{$12: 30-14: 00$} & \multirow[t]{4}{*}{ Factores claves de éxito } & Conceptos claves \\
\hline & & Trabajo individual y grupal \\
\hline & & Presentaciones \\
\hline & & Discusión grupal y acuerdos \\
\hline \multirow[t]{4}{*}{$15: 15-17: 00$} & \multirow{4}{*}{$\begin{array}{l}\text { Aspectos comunicacionales } \\
\text { en la relación }\end{array}$} & Afirmaciones y declaraciones \\
\hline & & Juicios y su estructura \\
\hline & & Fundamentación de los juicios \\
\hline & & Oferta - petición y promesa \\
\hline \multirow[t]{5}{*}{$17: 15-18: 30$} & \multirow[t]{5}{*}{ Estilos sociales } & Concepto de estilos sociales \\
\hline & & Asertividad/emotividad \\
\hline & & Autoevaluación de estilos sociales \\
\hline & & Características de cada estilo \\
\hline & & ¿Cómo relacionarnos con otros estilos? \\
\hline 18:30 - 19:00 & \multicolumn{2}{|l|}{ Cierre y próximos pasos } \\
\hline
\end{tabular}

finalidad de asegurar la calidad de las respuestas de los académicos encuestados y para evitar los sesgos que se pueden producir, no es posible realizar prueba t para nuestros dependientes. Para la comparación entre los años 2005 y 2007, se utilizó test $t$ de Student para nuestros independientes para comparar promedios y test chi-cuadrado para comparar proporciones. En general, las proporciones corresponden a la comparación de categonías de respuesta en rangos 4 y 5 de la encuesta. Se consideró significativo todo valor $\mathrm{p}$ inferior o igual a 0,05 . Todos los análisis estadísticos se hicieron usando el programa estadístico SPSS $16.0^{7-9}$. 


\section{RESUlTADOs}

En el estudio realizado el año 2005, respondieron la encuesta un total de 399 académicos (68\% del total de académicos) y el año 2007, 408 académi$\cos (68 \%)$. Ambos estudios contaron con la participación de todas las categorías académicas, y fueron representativos de 38 unidades académicas -incluida la Dirección de Escuela- lo cual nos permitió generalizar las conclusiones. Se calculó como indicador de validez el Alfa de Cronbach, el cual permitió medir la consistencia interna de las preguntas del instrumento, es decir la confiabilidad de cada ítem frente a la escala global. Los resultados fueron: Alfa de Cronbach año 2005 =0,962; Alfa de Cronbach año $2007=0,970$. Estos resultados pueden interpretarse como muy buenos, tomando en cuenta que valores de Alfa $>0,80$ se consideran de gran calidad; más aún, estos valores permiten plantear que sería posible eliminar algunas preguntas del instrumento, ya que con un número menor de preguntas no se perdería confiabilidad.

La percepción global de clima en la Escuela no mostró una mejoría significativa el año 2007 respecto de la evaluación 2005, situando su promedio en $68 \%$. Asimismo, en el último estudio $88 \%$ de las personas declaró estar de acuerdo o muy de acuerdo con la afirmación "tomando todo en consideración, me gusta trabajar en esta institución". Al comparar los resultados con la aplicación del año 2005, se observaron mejores puntuaciones en todas las dimensiones evaluadas. Las variables Comunicaciones (61\%), Liderazgo (72\%) y Condiciones Físicas (63\%) presentaron un alza durante los 2 últimos años y las dimensiones Estructura (77\%), Motivación (74\%) y Liderazgo (72\%) fueron percibidas más satisfactoriamente (Figura 1). Los factores Recompensa (55\%), Imparcialidad (60\%) y Condiciones Físicas (63\%) fueron evaluados más críticamente. Las jefaturas mostraron una percepción de Clima más positiva que los cargos no jefaturas en todas las dimensiones evaluadas. La mayor diferencia entre estos grupos se manifestó en la evaluación de las variables Estructura, Recompensa, Comunicaciones y Liderazgo. Desde el punto de vista de las categorías académicas, los Investigadores Asociados (84\%), fueron quienes mejor percibieron el clima laboral, y junto con ello mostraron un marcado incremento en su evaluación respecto del año 2005 (50\%). Le siguieron los Profesores Titulares (72\%) y los Profesores Asistentes $(70 \%)$, quienes mantuvieron la percepción demostrada por el estudio realizado el año 2005. Por el contrario, los Profesores Asociados Adjuntos mostraron una percepción promedio de clima más desfavorable (61\%), comparado con el estudio anterior (77\%). En cuanto a los Instructores Adjuntos (62\%) y los Profesores Asistentes Adjuntos, éstos mejoraron sus percepciones respecto de la evaluación anterior. En las Tablas 4 y 5 se presenta el estudio comparativo de las dimensiones en los

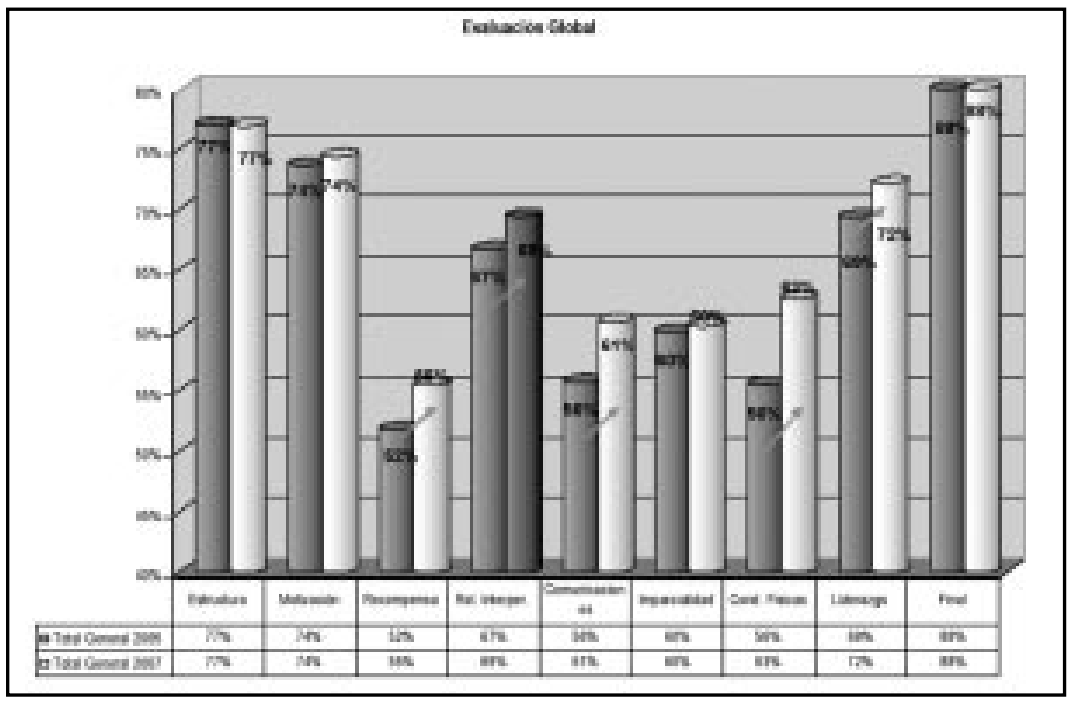

Figura 1. Resultados globales por dimensión estudiada en la encuesta. 
Tabla 4. Estudio comparativo de las dimensiones por grupo etáreo de los académicos. Año 2005

\begin{tabular}{|c|c|c|c|c|c|c|c|c|c|c|c|c|c|c|c|c|c|c|c|}
\hline \multirow[t]{2}{*}{ Dimensión } & \multicolumn{3}{|c|}{$<30$ años } & \multicolumn{3}{|c|}{30 a 40 años } & \multicolumn{3}{|c|}{40 a 50 años } & \multicolumn{3}{|c|}{50 a 60 años } & \multicolumn{3}{|c|}{60 a 65 años } & \multicolumn{3}{|c|}{$>65$ años } & \multirow[t]{2}{*}{ Valor $p$} \\
\hline & $\mathrm{N}$ & Media & DS & N & Media & DS & N & Media & DS & $\mathbf{N}$ & Media & DS & N & Media & DS & $\mathbf{N}$ & Media & DS & \\
\hline Estructura & 9 & 840 & ,284 & 129 & ,776 & ,235 & 127 & ,749 &, 257 & 73 & ,795 & ,244 & 26 & ,795 & ,251 & 7 & ,762 & 317 & ,758 \\
\hline Motivación & 9 & ,764 & 159 & 128 & ,761 & ,204 & 137 & 694 & 250 & 73 & ,771 & 224 & 26 & ,760 & ,255 & 8 & 781 & ,239 & 140 \\
\hline Recompensa & 9 & 611 & ,236 & 127 & ,497 & ,267 & 135 & ,488 & ,286 & 75 & ,567 & ,290 & 25 &, 540 & ,294 & 8 & ,563 & 198 & ,319 \\
\hline Interpersonales & 9 & ,778 & 199 & 126 & ,705 & 283 & 132 & ,605 & ,306 & 74 & ,723 & 271 & 26 & 642 & ,318 & 8 & ,725 & ,255 & ,029 \\
\hline Comunicación & 9 & 667 & ,265 & 128 &, 573 & ,279 & 133 &, 502 & ,332 & 75 &, 595 & ,290 & 26 &, 569 & ,308 & 8 &, 575 & ,292 & ,215 \\
\hline Imparcialidad & 9 & ,778 & ,233 & 127 & ,617 & ,278 & 135 &, 550 & ,327 & 74 & ,638 & 272 & 25 & 608 & ,319 & 7 &, 514 & ,344 & 103 \\
\hline Cond físicas & 9 &, 511 & ,376 & 128 &, 531 & ,366 & 135 &, 536 & ,382 & 75 & ,587 & ,370 & 26 & 600 & ,358 & 8 & 675 & ,320 & ,744 \\
\hline Liderazgo & 9 & ,711 & ,314 & 125 & ,709 & ,257 & 131 & 653 & ,322 & 73 & ,716 & 274 & 24 & ,763 & 289 & 8 & ,725 & ,255 & ,425 \\
\hline Escala PCTmas & 9 & ,727 & 198 & 120 & ,675 & 194 & 120 & ,624 & ,237 & 68 & ,702 & 210 & 24 & ,666 & ,238 & 6 & 701 & ,273 & 191 \\
\hline
\end{tabular}

Tabla 5. Estudio comparativo de las dimensiones por grupo etáreo de los académicos. Año 2007

\begin{tabular}{|c|c|c|c|c|c|c|c|c|c|c|c|c|c|c|c|c|c|c|c|}
\hline \multirow[t]{2}{*}{ Dimensión } & \multicolumn{3}{|c|}{$\measuredangle 0$ años } & \multicolumn{3}{|c|}{30 a 40 años } & \multicolumn{3}{|c|}{40 a 50 años } & \multicolumn{3}{|c|}{50 a 60 años } & \multicolumn{3}{|c|}{60 a 65 años } & \multicolumn{3}{|c|}{$>65$ años } & \multirow[t]{2}{*}{ Valor $\mathrm{p}$} \\
\hline & $\mathbf{N}$ & Media & DS & N & Media & DS & N & Media & DS & $N$ & Media & DS & $\mathbf{N}$ & Media & DS & $\mathrm{N}$ & Media & DS & \\
\hline Estructura & 10 & ,700 & ,323 & 137 & ,765 & ,263 & 123 & ,774 & 269 & 82 & ,760 & ,280 & 19 & 807 & ,264 & 14 & ,762 & ,261 & ,946 \\
\hline Motivación & 10 & ,688 & ,284 & 136 & ,744 & 220 & 131 & ,739 & ,228 & 85 & ,715 & ,266 & 20 & ,781 & ,206 & 14 & ,795 & 200 & ,710 \\
\hline Recompensa & 10 & ,417 & ,307 & 139 & ,538 & ,293 & 127 & ,518 & ,287 & 88 &, 583 & ,299 & 21 &, 563 & ,250 & 13 & 603 & ,323 & ,401 \\
\hline Interpersonales & 10 & ,700 & ,279 & 139 & ,730 & ,265 & 121 & ,675 & ,294 & 83 & ,684 & ,307 & 20 & ,735 & ,322 & 13 &, 515 & ,276 & 147 \\
\hline Comunicación & 10 &, 540 & ,327 & 136 & ,666 & 298 & 125 &, 571 & ,309 & 85 & ,5469 & ,279 & 21 &, 590 & ,293 & 14 & ,414 & ,328 & ,014 \\
\hline Imparcialidad & 10 &, 580 & ,346 & 137 & 601 & ,324 & 127 &, 576 & ,313 & 83 & ,610 & ,317 & 21 & ,648 & ,244 & 14 &, 543 & ,287 & ,892 \\
\hline Cond físicas & 10 & ,300 & ,316 & 140 & ,626 & ,364 & 128 &, 594 & ,353 & 87 & ,678 & ,375 & 20 & 690 & ,346 & 14 & ,671 & ,300 & ,038 \\
\hline Liderazgo & 10 & 690 & ,314 & 136 & ,752 & ,264 & 125 & 690 & ,300 & 85 & ,708 & ,274 & 19 & ,763 & ,295 & 13 &, 531 & ,312 & ,094 \\
\hline Escala PCTmas & 10 & ,612 & ,257 & 127 & ,696 & 214 & 106 & ,667 & ,238 & 74 & 680 & ,237 & 18 & ,723 & ,223 & 12 & ,597 & 223 & ,541 \\
\hline
\end{tabular}

dos años de estudio. Dentro de las dimensiones evaluadas, Comunicaciones ( $p=0,62)$ y Condiciones Físicas $(p=0,008)$ fueron las que presentaron un mayor aumento entre ambos períodos.

Al considerar la edad de los académicos, aquellos entre 60 y 65 años evaluaron más favorablemente las condiciones de su entorno laboral, seguidos por el grupo etáreo entre 30 y 40 años. El grupo de personas menores de 30 años, inversamente a los resultados encontrados el año 2005, percibieron el clima laboral de manera más desfavorable. En términos de género, las diferentes variables evaluadas obtuvieron en general una mejor apreciación por parte de los hombres (Figura 2). Entre los resultados obtenidos en la encuesta del año 2007 destaca la prioridad de temas a mejorar otorgada a nivel de Escuela al factor rentas (59\%), seguido por desarrollo académico (42\%) y muy de cerca formación integral de los académicos (37\%). A nivel de Departamentos, el factor desarrollo académico lideró las preferencias a mejorar (53\%), seguido por rentas (51\%) y sistema de incentivos asociados a metas (33\%) (ver Figuras 3a y 3b). Cabe señalar que la distribución de promedios a nivel de Departamento entregó una gran dispersión, por lo que se requiere un análisis más específico al interior de cada unidad académica; no obstante, las áreas con mayor apoyo fueron liderazgo y relaciones interpersonales. 


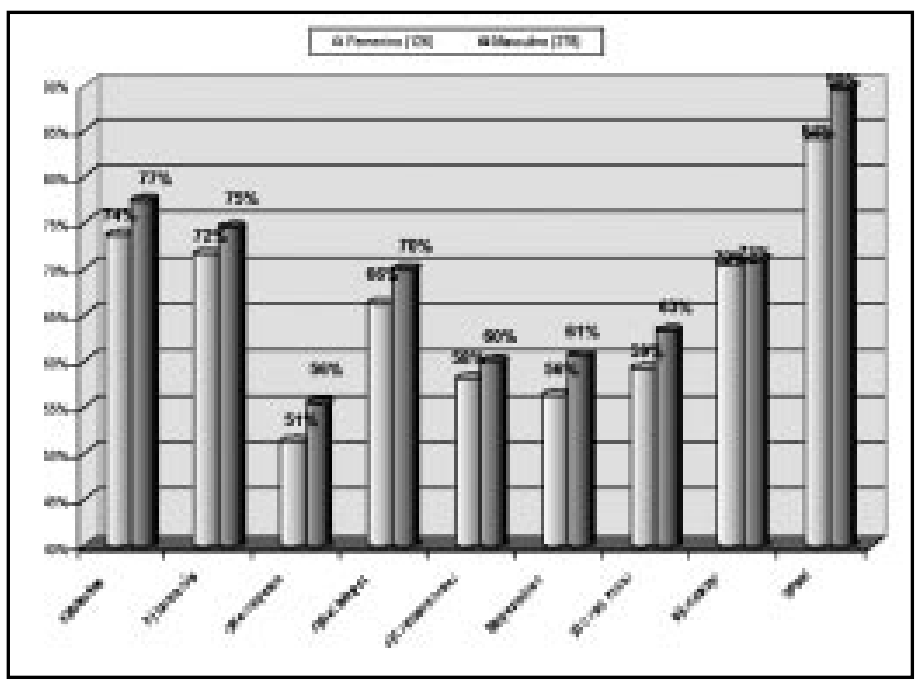

Figura 2. Resultados de las dimensiones estudiadas en la encuesta, evaluadas por género de los académicos.

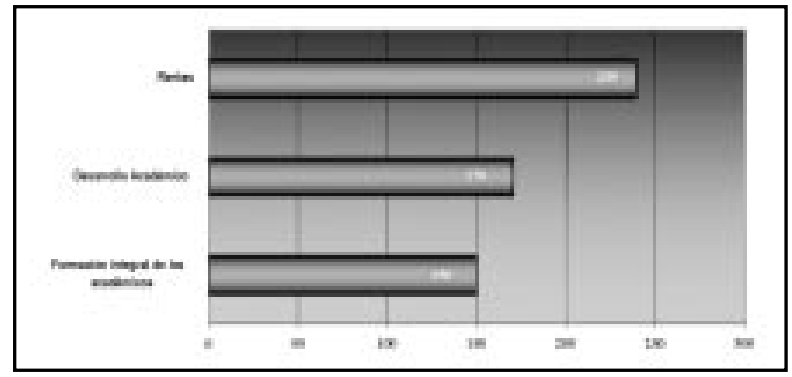

Figura 3a. Prioridades entregadas por los académicos, señalando los aspectos principales a solucionar en la escuela de medicina.

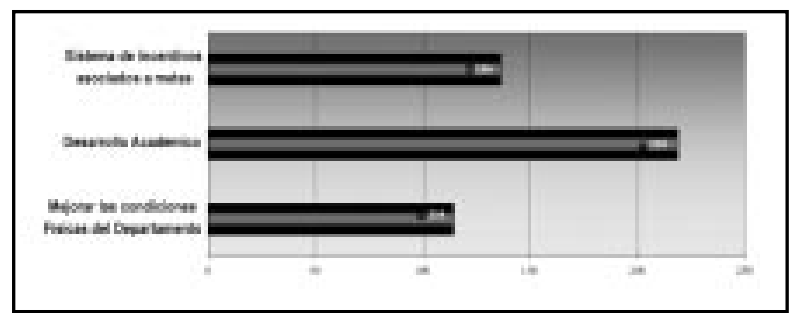

Figura 3b. Prioridades entregadas por los académicos, señalando los aspectos principales a solucionar en los departamentos académicos.

Durante el período 2005-2007 se realizaron distintas acciones de intervención, tanto a nivel global como específico, cuyo objetivo era mejorar la percepción en los aspectos más críticos y mantener las fortalezas en las áreas consideradas como tales. Múltiples investigaciones a través del tiempo indican que la presencia de determinadas prácticas de liderazgo correlacionan positivamente con una mejor percepción del clima laboral por parte de los participantes de un grupo. En este ámbito se implementaron las siguientes acciones: a) Seminario de Gestión Ejecutiva para Jefes de Departamento (programa de $40 \mathrm{~h}$ dirigido a todos los jefes de departamento sobre conocimientos básicos de administración; b) Programa de Prácticas Directivas (taller de $16 \mathrm{~h}$ dirigido a todos los jefes de departamento sobre desarrollo de habilidades básicas de liderazgo), y c) asesoría y acompañamiento individual (trabajo específico con jefaturas de apoyo y asesoría para el trabajo de equipo y resolución de conflictos). Como acción concreta durante el año 2006 se entregó un Manual de Beneficios a todos los académicos de la Facultad, el cual presentaba en forma detallada todos los beneficios y los procedimientos asociados a estos.

\section{DisCUSIÓN}

Los resultados de nuestro estudio demostraron las percepciones de los docentes de la Escuela de 
Tabla 6. Comparación 2005-2007 de promedios de cada dimensión y escala global

\begin{tabular}{|c|c|c|c|c|c|}
\hline Dimensión & Período & $\mathbf{n}$ & Media & Error estándar & $\begin{array}{l}\text { Significancia } \\
\text { estadística }\end{array}$ \\
\hline \multirow[t]{2}{*}{ Estructura } & 2005 & 386 & 7749 & ,01252 & \multirow{2}{*}{ 666 } \\
\hline & 2007 & 388 & ,7669 & ,01364 & \\
\hline \multirow[t]{2}{*}{ Motivación } & 2005 & 396 & ,7374 & ,01151 & \multirow{2}{*}{ 980 } \\
\hline & 2007 & 399 & 7378 & ,01162 & \\
\hline \multirow[t]{2}{*}{ Recompensa } & 2005 & 394 & ,5207 & 01417 & \multirow{2}{*}{,287 } \\
\hline & 2007 & 401 &, 5424 & , 01457 & \\
\hline \multirow[t]{2}{*}{ Interpersonales } & 2005 & 390 & ,6692 & , 01488 & \multirow{2}{*}{ 198 } \\
\hline & 2007 & 389 & ,6961 & ,01465 & \\
\hline \multirow[t]{2}{*}{ Comunicación } & 2005 & 393 &, 5593 & ,01518 & \multirow{2}{*}{,062 } \\
\hline & 2007 & 394 & ,5995 & 01521 & \\
\hline \multirow[t]{2}{*}{ Imparcialidad } & 2005 & 392 & ,6015 & ,01527 & \multirow{2}{*}{,782 } \\
\hline & 2007 & 395 &, 5954 & ,01578 & \\
\hline \multirow[t]{2}{*}{ Condiciones físicas } & 2005 & 396 & ,5571 & ,01854 & \multirow{2}{*}{,008 } \\
\hline & 2007 & 402 & ,6259 & ,01807 & \\
\hline \multirow[t]{2}{*}{ Liderazgo } & 2005 & 383 & 6982 & , 01472 & \multirow{2}{*}{ 418 } \\
\hline & 2007 & 391 & 7148 & ,01437 & \\
\hline \multirow[t]{2}{*}{ Escala global } & 2005 & 360 & ,6654 & ,01147 & \multirow{2}{*}{ 391 } \\
\hline & 2007 & 350 & 6798 & ,01220 & \\
\hline
\end{tabular}

Medicina en varias áreas de interés en su desarrollo académico y laboral. El estudio demostró además que el instrumento utilizado para medir el clima laboral en un período de 2 años es estable y útil.

En una serie recientemente publicada se establece que los cambios en el ambiente docente debido a las mayores exigencias por la demanda asistencial, han provocado un nivel de estrés importante, que afecta negativamente el plano personal y profesional, lo que estaría asociado al bienestar de los académicos ${ }^{6}$. En un interesante estudio en que se comparan cuatro escuelas de Estados Unidos de Norteamérica, en seis de los factores evaluados, un promedio de 390 docentes de ciencias básicas reportó un grado mayor de satisfacción laboral que los docentes clínicos. Algunos de estos factores fueron "recibir consejería académica", "capacidad de obtener gratificación personal de su trabajo", "tiempo asignado a la docencia" y "volumen de trabajo". En las cuatro escuelas, hubo una pequeña pero significativa correlación negativa entre la preocupación de los encuestados sobre la situación financiera institucional y el nivel de satisfacción personal y tensión laboral; y una correlación positiva con depresión y ansiedad. Entre los docentes de 15 especialidades que respondieron la encuesta, quienes se identificaron como cirujanos reportaron el mayor número de síntomas depresivos, el mayor nivel de ansiedad, el menor grado de satisfacción laboral y el mayor nivel de tensión laboral ${ }^{10}$. En un estudio reciente, hasta $42 \%$ de los docentes con frecuencia pensó en dejar la Facultad y uno de cada cinco "considera a menudo" la posibilidad de jubilar anticipadamente ${ }^{11}$. Los autores destacan la importancia de desarrollar estrategias de retención que incluyan el reconocimiento laboral y las perspectivas de desarrollo profesional y académico.

El tomar conciencia de la diversidad de valores y características generacionales hace po- 
sible que la Escuela de Medicina pueda identificar el origen de muchos de estos temas y desafíos y planificar las soluciones adecuadas, las que consideran un cambio en las políticas referentes al equilibrio trabajo y vida familiar, al uso de diversos tracks académicos con diferentes roles, a la contratación de académicos a tiempo parcial, a la creación de una variedad de programas de desarrollo académico orientada hacia diferentes necesidades, a la definición de recompensas e incentivos adecuados, y a la creación de reconocimientos de estos diferentes perfiles por parte de los pares. De esta forma, se puede mitigar el conflicto, promover la diversidad, y permitir a los equipos multigeneracionales funcionar de manera más efectiva y creativa en el ámbito de la educación, investigación y actividad asistencial ${ }^{12,13}$. Un aspecto que deberá observarse con más detención se refiere a la evolución de la percepción de clima que tienen las generaciones más jóvenes, en cuanto a si estamos frente a una situación puntual o frente a una problemática más profunda. El desafío es cómo preservar el ambiente y los aspectos positivos de la organización y al mismo tiempo dar respuesta a las expectativas de las generaciones de nuevos académicos, que presentan las motivaciones y aspiraciones propias de su generación.

Entre los médicos en formación, la relevancia asignada al clima laboral es importante. Entender el concepto que tienen los alumnos de medicina respecto de un ambiente óptimo de educación de postítulo puede ayudar a los docentes a estructurar de manera efectiva sus programas para entregar el equilibrio más efectivo entre educación, actividad asistencial y bienestar de los becados. La selección del programa parece ser un proceso complejo que involucra la consideración de muchos componentes, donde se destacan el rol del docente como modelo, un ambiente docente de postítulo efectivo que equilibra la docencia, el cuidado del paciente, y el bienestar de los residentes, que debiera ser un objetivo importante de los programas de postítulo ${ }^{14-17}$. En los estudios realizados al cuerpo académico de una escuela de Medicina, las mujeres y los hombres diferían considerablemente en sus percepciones sobre el género en diversos aspectos del clima laboral: trabajo en redes, ambiente profesional, obstáculos para el ascenso académico y razones para dejar la medicina académica. En general, las mujeres percibían con mayor frecuencia la existencia de una red informal que excluía a los académicos por el género18-19. En nuestro estudio hubo una menor percepción en todos los factores estudiados por parte de las docentes mujeres, lo que es un desafío de trabajo futuro.

Dentro de las primeras conclusiones que se puede observar con nuestro trabajo es que al realizar este tipo de mediciones hay implícito un mecanismo de intervención. Esta ocurre, en algunos casos, inmediatamente después de respondida la encuesta, mediante la implementación de medidas inmediatas, en otras y tal vez las más frecuentes, en una reunión de retroalimentación. En esa instancia se puede realizar un diagnóstico y acordar algunas medidas de cambio y en otros casos solicitar ayuda externa al departamento académico para profundizar el diagnóstico y acordar medidas más profundas ${ }^{20-21}$. También se pudo observar y focalizar los esfuerzos de intervención en tres niveles: institucional (a través de recompensas económicas), jefatura (por medio de refuerzo de prácticas directivas) y personal (implementando equipos de trabajo).

Este tipo de estudios son muy importantes para planificar estrategias de desarrollo de clima laboral, las que deben incluir diferentes aspectos, tales como: reconocimiento y estímulo profesional, beneficios académicos, estructuras de apoyo para un trabajo integral y apoyo a la educación continua y al perfeccionamiento académico. En especial, se debe trabajar en la cultura de la comunicación, franqueza, respeto e identificación institucional, que en el caso de nuestra Escuela de Medicina debe centrarse en el servicio a nuestros alumnos de pre y postgrado, y reflejarse en una atención orientada al servicio del paciente. Nuestro desafío es que esta línea de trabajo e investigación se perfeccione utilizando este instrumento que ha demostrado ser estable, eficaz y útil. A través de un seguimiento profesional de las percepciones y tendencias de los académicos y del apoyo con consejería académica y de liderazgo institucional, se pueda lograr un clima laboral que permita el mayor desarrollo humano y profesional de los docentes de esta comunidad académica. 


\section{REFERENCIAS}

1. Howell LP, Servis G, Bonham A. Multigenerational Challenges in Academic Medicine: UC Davis's Responses. Acad Med 2005; 80: 527-32.

2. SÁnchez I. Desafíos para el desarrollo académico de una Escuela de Medicina. Rev Méd Chile 2008; 136: 256-62.

3. Buckel J, Brown AJ. Generation X: Implications for faculty recruitment and development in academic health centers. Acad Med 2005; 80: 203-4.

4. Murray A, Montgomery JE, Chang H, Rogers WH, Inui T, SAFRAN DG. Doctor discontent: a comparison of physician satisfaction in different delivery system settings, 1986 and 1997. J Gen Intern Med 2001; 16: 452-9.

5. Deckard G, Meterko M, Field D. Physician burnout: an examination of personal, professional, and organizational relationships. Med Care 1994; 32: 745-54.

6. Demmy TL, Kivlahan C, Stone TT, Teague L, Sapienza P. Physician's perceptions of institutional and leadership factors influencing their job satisfaction at one academic medical center. Acad Med 2002; 77: 1235-40.

7. EveritT B, Howell D. Encyclopedia of statistics in behavioral science. Willey, vol 4, 2005.

8. Arritage P, Cotton T. Encyclopedia of biostatistics. Willey, 2nd Ed., 2005.

9. Gerber S, Voelkl K. Using SPSS for Windows. $2^{\text {nd }}$ Ed., Spinger 2005.

10. Schindler BA, Novack DH, Cohen DG, Yager J, Wang D, Shaheen NJ ET AL. The Impact of the Changing Health Care Environment on the Health and Well Being of Faculty at Tour Medical Schools. Acad Med 2006; 81; 27-34.

11. Lowenstein S, Fernández G, Crane L. Medical school faculty discontent: prevalence and predictors of intent to leave academic careers. BMC Med Educ 2007; 7:37. Published online 2007 October 14. doi: 10.1186/1472-6920-7-37.

12. Dorsey ER, Jarjoura D, Rutecki GW. Influence of controllable lifestyle on recent trends in specialty choice by US medical students. JAMA 2003; 290: 1173-8.

13. Rosenberg L Physician-scientists: endangered and essential. Science 1999; 283: 331-2.

14. NuthalaPaty FS, Jackson JR, Owen J. The Influence of Quality-of-Life, Academic, and Workplace Factors on Residency Program Selection. Acad Med 2004; 79: 417-25.

15. Shanafelt T, Habermann T. Medical residents' emotional well-being. JAMA 2002; 288: 1846-7.

16. RIDER EA. How to arrange a shared or part-time residency. J Am Med Women's Assoc 1992; 47: 138-9.

17. Biand KI, Isaacs G. Contemporary trends in student selection of medical specialties: the potential impact on general surgery. Arch Surg 2002; 137: 259-67.

18. Foster SW, McMurray JE, Linzer M, Leavitt JW, Rosenberg M, Carnes M. Results of a Gender-climate and Work-environment Survey at a Midwestern Academia Health Center. Acad Med 2000; 75: 653-60.

19. McPhiшips HA, Burke AE, Sheppard K, Pallant A, Stapleton B, Stanton B. Toward Creating FamilyFriendly Work Environments in Pediatrics: Baseline Data From Pediatric Department Chairs and Pediatric Program Directors. Pediatrics 2007; 119: e596e602.

20. Rainieri A. Estilos de Dirección como determinantes del Clima Laboral en Chile. Rev ABANTE 2006; 9: 333.

21. Fox G, Schwartz A, Hart KM. Work-family balance and academic advancement in medical schools. Acad Psychiatry 2006; 30: 227-34. 